\title{
Green Polymers toward Nanobiotechnology(I): Synthesis of Glycopolypeptides and Their Analogues
}

\author{
Zhao Wang, ${ }^{a}$ Ana Rute Neves, ${ }^{b}$ Filipe Olim, ${ }^{b}$ Helena Tomás, ${ }^{b}$ Shi Tang, ${ }^{*, c}$ and Ruilong Sheng ${ }^{\star, b}$ \\ ${ }^{a}$ Department of Materials, Jinling Institute of Technology, Nanjing, Jiangsu 211169, China \\ ${ }^{b}$ CQM-Centro de Química da Madeira, Universidade da Madeira, Campus da Penteada, \\ 9000-390 Funchal, Portugal \\ ${ }^{c}$ College of Chemistry and Chemical Engineering, Jishou University, Jishou, Hunan 416000, China
}

Email: ruilong.sheng@staff.uma.pt (R. S.); Tel: +351-291705254

\begin{abstract}
Harnessing natural-based renewable molecular resources to construct functional synthetic green polymers is a promising research frontier at the interface of sustainable/green chemistry, polymer chemistry and nanobiotechnology. As natural glycoprotein mimics/analogues and biocompatible building blocks of nanobiomaterials, synthetic functional glycopolypeptides and their structural/functional analogues have attracted great attentions in recent years. This mini-perspective article reviewed current synthetic strategies and methods of glycopolypeptides and their analogues. The pros and cons of the synthesis protocols were discussed, moreover, possible future perspectives in this field were also stated.
\end{abstract}

Keywords glycopolypeptide, green polymer, synthesis, perspective

Glycopolypeptides, are generally referred as polypeptides containing pendant sugar moieties, sometimes glycopeptide analogs such as chemical conjugates of polyamino acids/polypeptides with polysaccharides/glycopolymers, are also referred to glycopolypeptides. ${ }^{[1]}$ As natural glycoprotein mimics, synthetic glycopolypeptides are able to be employed as artificial models to decipher the structures (such as $\alpha$-helix, $\beta$-sheet and advanced architectures) and functions (such as aggregation, molecular recognition, cell adhesion, and signal transduction) of their natural glycopeptide/glycoprotein counterparts. ${ }^{[2]}$ Moreover, they could also be utilized as biocompatible building blocks for construction of biocompatible, biodegradable, morphology-varied and function-diversified nano-assemblies/aggregates as functional platforms towards biomedical applications. ${ }^{[3]}$

To prepare functional glycopolypeptide and their analogues, to date, many synthetic strategies and functionalization/modification methods have been developed. ${ }^{[4]}$ In general, the synthetic strategies of glycopolypeptides could be divided into three categories (Figure 1): (a) Synthesis of glycopolypeptides by post-polymerization glycosylation of synthetic polypeptides. Glycosides or sugar-containing moieties were grafted onto polyamino acid side chain/polypeptide backbones via chemical coupling reactions (activated ester coupling, isothiocyanide linking, click chemistry, thiol-ene/yne addition, methionine alkylation, and so on). ${ }^{[5]}$ Among them, thiol-ene/yne and methionine alkylation methods possess very high (near quantitative) grafting reactivity ${ }^{[6]}$ Nevertheless, in the "grafting to" approach, the grafting efficiency of sugar-containing moieties largely depend on the key factors such as molecular hydrophobicity/ philicity, steric hindrance, reaction mediums and catalysts, which remain still challenges to be addressed; (b) Synthesis of glycopolypeptides by ring-opening polymerization (ROP) of glycosylated $\mathrm{N}$-carboxyanhydride (glyco-NCA) monomers. The ROP approach to functional glycopolypeptide from functional NCA monomers can effectively avoid the drawback of incomplete coupling/grafting reaction in post-polymerization glycosylation. ${ }^{[7]}$ Many glyco-NCA monomers were synthesized and

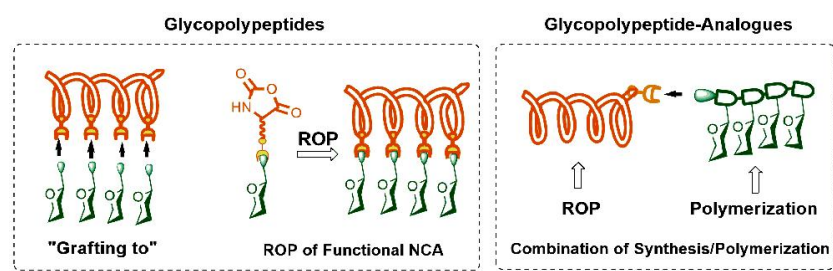

Figure 1 Current synthetic strategies of glycopolypeptides and their analogues.

successfully applied to the preparation of subsequent glycopeptides, ${ }^{[8]}$ whereas the preparation and purification of many high polarity glycol-NCA monomers are hard-to-manipulate. Therefore, it is highly desirable to: 1 . developing stable and easy-to-handle glycol-NCA monomers by choosing proper protection moieties and expanding the moieties' diversity; 2 . using efficient and easy-to-operate organic/polymer synthetic (e.g., "one-pot" or cascade glycosylation) methods, which need to be highly focused in future research; (c) Synthesis of glycopolypeptide analogues by glycosylation at the termini of polypeptides. In order to overcome the cumbersome preparation and purification process for glycopolypeptides and to expand the structural diversity, some glycopolypeptides analogues were developed by coupling glycopolymer to the termini of polyamino acid/polypeptide via efficient "Click" reaction in recent years. By this means, monosaccharide, oligosaccharide and polysaccharide-containing glycopolymers can be effectively coupled to the end of polyamino acid/polypeptide chains, to obtain linear, branched and dendritic glycopolypeptides and expand the structural diversity. ${ }^{[9]}$ The key issue in this area is the rational design of monomers, controlling the reactivity and polymer polydispersity, adaptivity of the block copolymers and linkage between each blocks. ${ }^{[10]}$ Notably, efficient combination of multiple reactions (ROP, controllable free radical polymerization, Click coupling, etc.) need to be further developed to prepare glycopolypeptide analogues. ${ }^{[11]}$ These structural-diversified glycopolypeptide analogues may serve as new artificial 


\section{Perspective}

models for the study of glycomics, bio-recognition/-adhesion, nano-biomaterials/theranostics, ${ }^{[12]}$ as well as 3D-printable matrix for cell/tissue engineering.

Regarding the future of synthetic glycopolypeptide and their analogues, there are still vast spaces for extensive research and development. The following aspects need to be emphasized (Figure 2): (1) simplifying the reaction/synthesis steps and developing efficient purification strategies for the preparation of glycopolypeptides; developing green synthesis/polymerization processes by using natural-based renewable building blocks, environmental friendly reaction mediums (water, ionic liquid), and heavy metal-free catalytic processes (photocatalysis, organocatalysis and enzyme catalysis), solid-phase polypeptide synthesis may need to be considered, (2) expanding the topological diversity of the glycopolypeptides structures (such as hyperbranched structures, advanced symmetric/asymmetric structures, macrocyclic structures, as well as special 3D geometry such as Janus, Knots and Origami); (3) developing "smart" functional (stimuli-responsive, self-regulated/adaptive, as well as multifunction-intergrated) glycopolypeptides and analogues towards the requirement of precision and personalized medicine; (4) design and synthesis of biofunction (such as bio-recognition and receptor targeting, signal transduction and molecular chaperone-simulating)-oriented glycopolypeptides to mimic the natural glycoproteins/glycopolypeptides; ${ }^{[13]}$ (5) studying and elucidating the structure-function relationship (SFR) between the structures of glycopolypeptides and their physico-chemical/biological functions.
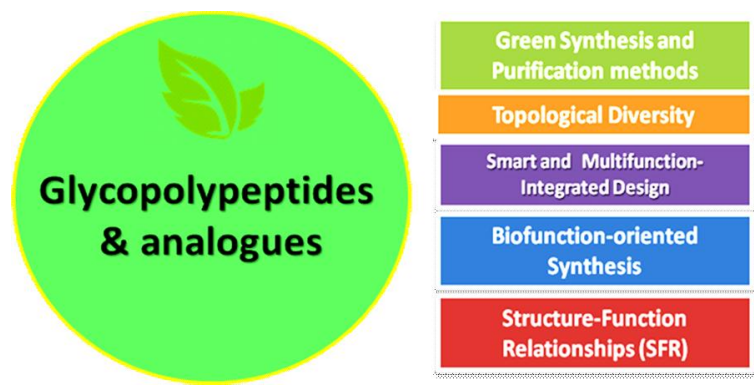

Figure 2 Future perspective of synthetic glycopolypeptide and their analogues.

By using the synthesized functional glycopolypeptide as building blocks, further incorporating (self or forced) supramolecular assembly and controllable nanotechnology, ${ }^{[14]}$ new series of high performance glycopolypeptide nanoassemblies could be created. We can anticipate that, these "green, smart and sweet" functional biomimetic nanoassemblies could serve as sustainable and controllable nanoplatforms towards future biomedical applications.

\section{Acknowledgement}

We appreciate ARDITI-Agência Regional para o Desenvolvimento da Investigação Tecnologia e Inovação, through the project M1420-01-0145-FEDER-000005-Centro de Química da Madeira-CQM ${ }^{+}$(Madeira 14-20), ARDITI-2017ISG-003 and ARDITI-2017-PDG-011, FCT-Fundação para a Ciência e a Tecnologia (project PEst-OE/QUI/UI0674/2019, CQM, Portuguese Government funds), and project PROEQUI PRAM-Reforço do Investimento em Equipamentos e Infraestruturas Científicas na RAM (M1420-01-0145-FEDER000008) for sponsorship. Dr. Zhao Wang thanks the foundation support from Jinling Institute of Technology (Project No: jit-b201828). Dr. Shi Tang thanks the National Natural Science Foundation of China (Nos. 21662013 and 21462017).

\section{References}

[1] (a) Bonduelle, C.; Lecommandoux, S. Biomacromolecules 2013 14, 2973.; b) Krannig, K. S.; Schlaad, H. Soft Matter 2014, 10, 4228.

[2] (a) Miura, Y. Polym. J. 2012, 44, 679; (b) Kiessling, L. L.; Grim, J. C. Chem. Soc. Rev. 2013, 42, 4476; (c) Bonduelle, C.; Oliveira, H.; Gauche, C.; Huang, J.; Heise, A.; Lecommandoux, S. Chem. Commun. 2016, 52, 11251.

[3] Xiao, C. S.; Ding, J. X.; He, C. L.; Chen, X. Acta Polym. Sinica 2018, 1, 45.

[4] (a) Kramer, J. R.; Deming, T. J. Polym. Chem. 2014, 5, 671; (b) Deming, T. J. Chem. Rev., 2016, 116, 786.

[5] (a) Gauche, C.; Lecommandoux, S. Polymer 2016, 107, 474; (b) Rhodes, A. J.; Deming, T. J. ACS Macro Lett. 2013, 2, 351; (c) Yang, H. K.; Bao, J. F.; Mo, L.; Yang, R. M.; Xu, X. D.; Tang, W. J.; Lin, J. T.; Wang, G. H.; Zhang, L. M.; Jiang, X. Q. RSC Adv. 2017, 7, 21093; (d) Krannig, K. S.; Huang, J.; Heise, A.; Schlaad, H. Polym. Chem. 2013, 4, 3981; (e) Kramer, J. R.; Deming, T. J. Biomacromolecules 2012, 13, 1719.

[6] (a) Kramer, J. R.; Deming, T. J. Chem. Commun. 2013, 49, 5144; (b) Krannig, K. S.; Doriti, A.; Schlaad, H. Macromolecules 2014, 47, 2536.

[7] Kramer, J. R.; Deming, T. J. J. Am. Chem. Soc. 2010, 132, 15068.

[8] (a) Kramer, J. R.; Deming, T. J. J. Am. Chem. Soc. 2012, 134, 4112; (b) Krannig, K. S.; Doriti, A.; Schlaad, H. Macromolecules 2014, 47, 2536.

[9] (a) Bonduelle, C.; Mazzaferro, S.; Huang, J.; Lambert, O.; Heise, A.; Lecommandoux, S. Faraday Discuss. 2013, 137; (b) Mohamed Wali, A. R.; Zhou, J.; Ma, S.; He, Y.; Yue, D.; Tang, J. Z.; Gu, Z. Int. J. Pharm. 2017, 525, 191; (c) Pranantyo, D.; Xu, L. Q.; Hou, Z.; Kang, E. T.; Chan-Park, M. B. Polym. Chem. 2017, 8, 3364.

[10] (a) Upadhyay, K. K.; Meins, J.-F. Le; Misra, A.; Voisin, P.; Bouchaud, V.; Ibarboure, E.; Schatz, C.; Lecommandoux, S. Biomacromolecules 2009, 10, 2802; (b) Yang, H. K.; Zhang, L.-M. M. Mater. Sci. Eng. C. Mater. Biol. Appl. 2014, 41, 36.

[11] (a) Fu, L.; Sun, C.; Yan, L. ACS Appl. Mater. Interfaces 2015, 7 2104; (b) Wang, Z.; Sheng, R.; Luo, T.; Sun, J.; Cao, A. Polym. Chem. 2017, 8, 472

[12] (a) Wang, Z.; Luo, T.; Sheng, R.; Li, H.; Sun, J.; Cao, A. Biomacromolecules 2015, 17, 98; (b) Sun, J.; Sheng, R.; Luo, T.; Wang, Z.; Li, H. Cao, A. J. Mater. Chem. B. 2016, 4, 4696; (c) Wang, Z.; Luo, T.; Cao, A.; Sun, J.; Jia, L.; Sheng, R. Nanomaterials 2018, 8, 136

[13] (a) Zhou, M.; Delaveris, C.; Kramer, J.; Kenkel, J.; Engleman. E.; Bertozzi, C. Angew. Chem. Int. Ed. 2018, 57, 3137; (b) Pandey, B.; Patil, N.; Bhosle, G.; Ambade, A.; Gupta, S. Bioconjugate Chem. 2019, 30, 633 .

[14] (a) Pati, D.; Das, S.; Patil, N. G.; Parekh, N.; Anjum, D. H.; Dhaware, V.; Ambade, A. V.; Sen Gupta, S. Biomacromolecules 2016, 17, 466; (b) Liu, Y.; Zhang, Y.; Wang, Z.; Wang, J.; Wei, K.; Chen, G.; Jiang, M. J. Am. Chem. Soc. 2016, 138, 12387; (c) Machado, C.; Smith, I.; Savin, D. Macromolecules 2019, 52, 1899.

Received April 4, 2019 Accepted April 30, 2019 